\title{
Twelve Frequently Asked Questions About Growth Curve Modeling
}

\author{
Patrick J. Curran, Khawla Obeidat, and Diane Losardo \\ University of North Carolina at Chapel Hill
}

\begin{abstract}
Longitudinal data analysis has long played a significant role in empirical research within the developmental sciences. The past decade has given rise to a host of new and exciting analytic methods for studying between-person differences in within-person change. These methods are broadly organized under the term growth curve models. The historical lines of development leading to current growth models span multiple disciplines within both the social and statistical sciences, and this in turn makes it challenging for developmental researchers to gain a broader understanding of the current state of this literature. To help address this challenge, the authors pose 12 questions that frequently arise in growth curve modeling, particularly in applications within developmental psychology. They provide concise and nontechnical responses to each question and make specific recommendations for further readings.
\end{abstract}

\begin{abstract}
A foundational goal underlying the developmental sciences is the systematic construction of a reliable and valid understanding of the course, causes, and consequences of human behavior. Consistent with this goal, longitudinal studies have long played a critically important role in developmental psychology, and these designs are becoming increasingly common in contemporary research practices. However, consistent with the old adage $b e$ careful for what you ask - you might just get it, once longitudinal data are obtained, they must then be thoughtfully and rigorously analyzed. And as any developmental researcher can attest, statistical models for longitudinal data can become exceedingly complex exceedingly quickly, both in terms of fitting models to data and properly interpreting results with respect to theory (e.g., Curran \& Willoughby, 2003; Nesselroade, 1991; Wohlwill, 1991). Further, during the past decade, a host of powerful analytic methods have been developed that allow for the empirical evaluation of theoretically derived research hypotheses in ways not previously possible. Given the rapid onslaught of new methods, it can often be a significant challenge for researchers to stay abreast of ongoing developments and to incorporate these new techniques into their own programs of research. As quantitative psychologists who conduct substantive programs of research, we feel these very same pressures ourselves.
\end{abstract}

In an attempt to help organize the constantly shifting sands of new information, we have posed 12 specific questions that frequently arise with respect to growth curve modeling. We are under tight space constraints, so our rather modest intent is to provide brief and nontechnical responses to these questions and to recommend specific resources for further reading. The questions we pose are by no means exhaustive nor are our associated responses. Importantly, given our quest for brevity, we offer only a subset of available citations; the inclusion of one citation at the expense of another should be taken to mean 
nothing more than that we ran out of space. We hope that our brief foray through the intriguing yet sometimes bewildering topic of growth modeling might entice readers to consider ways in which these approaches might be incorporated into your own program of research. So let's give it a go.

\section{WHAT IS GROWTH CURVE MODELING?}

Growth curve modeling is a broad term that has been used in different contexts during the past century to refer to a wide array of statistical models for repeated measures data (see Bollen, 2007, and Bollen \& Curran, 2006, pp. 9-14, for historical reviews). However, within the past decade or so, this term has primarily come to define a discrete set of analytical approaches, particularly as applied within the social sciences. More specifically, the contemporary use of the term growth curve model typically refers to statistical methods that allow for the estimation of inter-individual variability in intra-individual patterns of change over time (e.g., Bollen \& Curran, 2006; Browne \& du Toit, 1991; McArdle, 2009; Preacher, Wichman, MacCallum \& Briggs, 2008; Raudenbush \& Bryk, 2002, pp. 160-204; Singer \& Willett, 2003). In other words, growth models attempt to estimate between-person differences in within-person change. Often these within-person patterns of change are referred to as time trends, time paths, growth curves, or latent trajectories. These trajectories might take on a variety of different characteristics that vary from person to person: They might be flat (i.e., showing no change over time), they might be systematically increasing or decreasing over time, and they might be linear or curvilinear in form. In many applications, the trajectories are the primary focus of analysis, whereas in others, they may represent just one part of a much broader longitudinal model.

The most basic growth model is composed of the fixed and random effects that best capture the collection of individual trajectories over time. Loosely speaking, a fixed effect represents a single value that exists in the population (e.g., the population mean height for men), and a random effect represents the random probability distribution around that fixed effect (e.g., the population variance in height for men). Consistent with these definitions, in the growth model, the fixed effects represent the mean of the trajectory pooling of all the individuals within the sample, and the random effects represent the variance of the individual trajectories around these group means. For example, for a linear trajectory, the fixed effects are estimates of the mean intercept (i.e., starting point) and mean slope (i.e., rate of change) that jointly define the underlying trajectory pooling of the entire sample; in contrast, the random effects are estimates of the between-person variability in the individual intercepts and slopes. Smaller random effects (i.e., smaller variances of intercepts and slopes) imply that the parameters that define the trajectory are more similar across the sample of individuals; at the extreme situation where the random effects equal 0 , all individuals are governed by precisely the same trajectory parameters (i.e., there is a single trajectory shared by all individuals). In contrast, larger random effects (i.e., larger variances of intercepts and slopes) imply that there are greater individual differences in the magnitude of the trajectory parameters around the mean values; that is, some individuals are reporting higher or lower intercepts, or steeper or less-steep slopes relative to others. Taken together, the fixed and random effects capture the general characteristics of growth for both the group as a whole and for the individuals within the group.

\section{HOW DO GROWTH MODELS DIFFER FROM MORE TRADITIONAL LONGITUDINAL MODELS?}

There is a long and rich history in the analysis of repeated measures data, and many methods have been proposed for use within the social sciences. Key traditional approaches include repeated measures analysis of variance and multivariate analysis of variance, as well as 
various methods for analyzing raw and residualized change scores (see Hedeker \& Gibbons, 2006, chaps. 2 and 3, for a review). The history of these methods has at times been quite contentious with strongly worded recommendations supporting or refuting particular approaches (e.g., Cronbach \& Furby, 1970; Rogosa, 1980; Rogosa \& Willett, 1985). Despite the disagreements over the use of one approach over another, growth models differ from traditional methods in several key respects. Most importantly, current approaches to growth modeling are highly flexible in terms of the inclusion of a variety of complexities including partially missing data, unequally spaced time points, non-normally distributed or discretely scaled repeated measures, complex nonlinear or compound-shaped trajectories, time-varying covariates (TVCs), and multivariate growth processes. All of these issues routinely arise in developmental research, yet all present significant challenges within traditional analytic approaches. Further, both analytical and simulation results show that growth models are typically characterized by much higher levels of statistical power than comparable traditional methods applied to the same data (e.g., B. O. Muthén \& Curran, 1997). To stress, traditional methods for analyzing repeated measures data remain a powerful tool in many research applications when the underlying assumptions are met. However, these methods become increasingly limited under conditions commonly encountered in social science research, whereas growth models typically are not.

\section{HOW ARE GROWTH MODELS FIT TO DATA?}

There are two general approaches used to fit growth models to observed data that share certain similarities but are also characterized by certain distinct differences (e.g., Bauer, 2003; Curran, 2003; Raudenbush, 2001; Willett \& Sayer, 1994). The first approach is to fit the growth model within the multilevel modeling framework (Bryk \& Raudenbush, 1987; Raudenbush \& Bryk, 2002; Singer \& Willett, 2003). The multilevel model was originally developed to allow for the nesting of multiple individuals within a group, such as children nested within classroom or siblings nested within family. However, the model can equivalently be applied to multiple repeated measures nested within each individual that allows for the direct estimation of a variety of powerful and flexible growth models. The second approach is to fit the growth model within the structural equation modeling (SEM) framework (e.g., Bollen \& Curran, 2006; Duncan, Duncan, \& Strycker, 2006; McArdle, 1988; McArdle \& Epstein, 1987; Meredith \& Tisak, 1990). The SEM incorporates the observed repeated measures as multiple indicators on one or more latent factors to characterize the unobserved growth trajectories. In many situations, the multilevel and SEM approaches to growth modeling are numerically identical, yet in others, there are important differences. For example, the multilevel model naturally expands to estimate higher levels of nesting (e.g., repeated measures nested within child, and child nested within classroom); the SEM approach is currently more limited in these situations. In contrast, the SEM is well suited to the estimation of latent variables that estimate and remove the effects of measurement error that might exist in the predictors or the outcomes; the multilevel model is currently more limited with respect to the estimation of comprehensive measurement models. However, the similarities between the multilevel and SEM approaches often outweigh the differences, and the optimal approach should be selected as a function of the particular research application at hand (Raudenbush, 2001).

\section{WHAT ARE THE DATA REQUIREMENTS TO USE GROWTH MODELS?}

Although there are few strict requirements for the types of data that might be analyzed using growth models, there are a number of general data characteristics that are particularly amenable to these methods. First, an adequate sample size is needed to reliably estimate growth models. However, what constitutes "adequate" cannot be unambiguously stated, because this depends in part on other characteristics of the research design (e.g., complexity 
of the growth model, amount of variance explained by the model). For example, growth models have successfully been fitted to samples as small as $n=22$ (Huttenlocher, Haight, Bryk, Seltzer, \& Lyons, 1991), although sample sizes approaching at least 100 are often preferred. Further, there is a close relation between the number of individuals and the number of repeated observations per individual (e.g., B. O. Muthén \& Curran, 1997); as such, the total number of person-by-time observations plays an important role in model estimation and statistical power as well. Second, growth models typically require at least three repeated measures per individual, although this requirement is also rather vague. For example, in the presence of partially missing data, some individuals might have just one or two observations, whereas others have three or more. However, three repeated measures over-identifies a linear trajectory (that is, there is more observed information than estimated information) and is thus preferred for at least a sizeable portion of the cases. Third, for the typical method of estimation called maximum likelihood (ML), it is assumed that the repeated measures are continuous and normally distributed. However, alternative methods of estimation allow for measures that are continuous and non-normally distributed (Satorra, 1990) or even discretely or ordinally scaled (e.g., Mehta, Neale, \& Flay, 2004). In sum, growth models may be fitted to many types of sample data structures, although care must be taken in the selection of proper models and methods of estimation that maximally correspond to the characteristics of the given data set.

\section{CAN GROWTH MODELS BE ESTIMATED WITH PARTIALLY MISSING DATA?}

Growth models can be estimated in the presence of partially missing data, although certain assumptions regarding the mechanism of "missingness" must be invoked for valid results. There are two general approaches to estimating models with partially missing data (Allison, 2001; Schafer, 1997; Schafer \& Graham, 2002). The first is direct ML (Arbuckle, 1996; Little \& Rubin, 1987). Under direct ML, the growth model is estimated by summing the individual contributions of each case such that observations with a larger number of data points are weighted more heavily than observations with a smaller number of data points. The second approach is called multiple imputation, and the growth model is estimated in a two-stage sequence (Rubin, 1987; Schafer, 1999). In the first stage, the missing data points are imputed based upon the characteristics of the non-missing data points, and this is done multiple times (typically 5 to 10 times). In the second stage, the growth model is fitted separately to each of the imputed data sets, and the results are pooled into a final set of estimates. Although extremely flexible, both approaches invoke explicit assumptions about the nature of missing data. Specifically, the missing data must be characterized as missing completely at random (e.g., cases are truly missing at random) or missing at random (e.g., cases are missing as a function of measured characteristics such as gender or ethnicity). Importantly, data that are missing not at random (e.g., cases are missing as a direct function of unmeasured characteristics such as the very value that is missing) cannot be included in standard growth modeling applications, and much more complex procedures are required (e.g., Heckman, 1976; Rubin, 1988).

\section{WHAT ARE THE DIFFERENT SHAPES OF GROWTH CURVES THAT CAN BE MODELED?}

A critically important first step in any growth model is the identification of the optimal functional form of the trajectory over time; that is, it must be established exactly how the repeated measures change as a function of time. If the incorrect functional form is used as the basis for the initial growth model, then expanding this model to include complexities such as predictors of growth or multiple group analysis will likely lead to biased results. The 
most basic form of growth is a random intercept-only model; this implies that there is a stable overall level of the repeatedly measured construct over time and individuals vary randomly around this overall level at any given time point. It may seem an oxymoron to call an intercept-only model "growth," but this is consistent with the notion of a trajectory that is simply flat with respect to time. This intercept-only model can then be expanded in a variety of directions. The most straightforward method is to consider the family of polynomial functions; examples include a straight line, a quadratic curve, and a cubic curve.

Polynomials are widely used given that these can be easily estimated within either the SEM or multilevel frameworks. Other more complex functional forms are possible including entire families of interesting exponential trajectories (e.g., monomolecular, logistic; Cudeck \& Harring, 2007). However, a variety of complications arise when incorporating these types of trajectories, because the parameters enter the model nonlinearly, making model estimation substantially more difficult, if not at times impossible. A flexible alternative is to use piecewise linear modeling to approximate complex nonlinear functions in which two or more linear trajectories are joined together to correspond to a potentially intractable nonlinear function (Bollen \& Curran, 2006, pp. 103-106; Raudenbush \& Bryk, 2002, pp. 178-179; Singer \& Willett, 2003, pp. 207-208). A final option is a fully latent curve model available within the SEM framework in which some or all of the loadings on the slope factor are freely estimated so that change optimally corresponds to the unique characteristics of the data under study (McArdle, 1988; Meredith \& Tisak, 1990).

\section{HOW IS THE ADEQUACY OF FIT FOR GROWTH MODELS ASSESSED?}

It is as essential to establish the adequate fit of the hypothesized model within the growth modeling framework as it is in any other statistical model (but see Coffman \& Millsap, 2006, for an alternative perspective). How this is best done directly depends upon the specific analytic strategy used to estimate the growth models. Within the SEM, it is possible to judge the fit of a hypothesized model relative to a saturated baseline model allowing for the estimation of standalone indices of overall fit for a given model. Examples include the model chi-square test statistic and fit indices such as the RMSEA (root mean squared error of approximation), CFI (comparative fit index), and TLI (Tucker-Lewis index), among many others. Within the multilevel framework, it is not possible to estimate a saturated baseline model to which to compare the hypothesized model. As such, there are no standalone measures of overall fit for a hypothesized model (although other indices of appropriate fit can be used such as residuals and Wald tests). Instead, comparisons of competing alternative models are required (which we believe is a strategy that could be used to a much greater extent within the SEM framework). If two comparison models are nested (i.e., if the parameters of one model are a direct subset of the parameters of the second model), then formal likelihood ratio tests can be calculated based on the differences between model deviance (see, e.g., Raudenbush \& Bryk, 2002, pp. 283-284). For models that are not nested, informal comparisons can be made using indices such as the Bayesian Information Criterion or the Akaike Information Criterion to rank order models (e.g., Bollen \& Long, 1993). Regardless of approach, it is extremely important that clear evidence be presented that supports the adequacy of fit of the hypothesized model to the observed data prior to drawing theoretical inferences from the results.

\section{HOW CAN PREDICTORS BE INCORPORATED INTO THE GROWTH MODEL?}

Once the optimal baseline growth model has been established, this can then be expanded to include one or more predictors of growth. The inclusion of predictors in the model results in what is often called a conditional growth model because the fixed and random effects are now "conditioned on" the predictors. There are generally two types of predictors to 
consider: time-invariant covariates (TICs) that do not change in value as a function of time and TVCs that at least in principle can change as a function of time. TICs typically predict the random components of growth directly with the goal of determining what variables are associated with individuals who report higher versus lower intercepts or steeper versus flatter slopes. For example, say that a linear trajectory is deemed to be the optimal functional form over time, and there is evidence of significant random effects in both the intercept and slope components of the trajectory. TICs can then be incorporated to predict this random variability in starting point and rate of change. This would directly evaluate hypotheses about whether characteristics of the individual (e.g., gender, treatment condition) are predictive of higher or lower starting points or steeper or less steep rates of change over time (e.g., Curran, Bauer \& Willoughby, 2004).

Importantly, TICs are assumed to be independent of the passage of time. In other words, the given value of the TIC could in principle be assessed at any time point as this is constant over time. This assumption is sometimes strictly true (e.g., biological sex, country of origin), and at other times, the construct might in principle vary with time but is only assessed at a single time period (e.g., baseline anxiety or initial reaction time). However, growth models can easily be expanded to include the effects of covariates that do vary as a function of time; these are TVC models (Bollen \& Curran, 2006, pp. 192-198; Raudenbush \& Bryk, 2002, pp. 179-186; Singer \& Willett, 2003, pp. 159-188). Whereas TICs directly predict the growth factors (e.g., Bollen \& Curran, 2006, Figure 5.1), TVCs directly predict the repeated measures while controlling for the influence of the growth factors (e.g., Bollen \& Curran, 2006, Figure 7.1). Thus, any given repeated measure is jointly determined by the underlying growth factors and the impact of the TVC at that time period. The TVC model can then be expanded to include interactions between the TVCs and time (to assess differences in the magnitude of the TVC effect as a function of time) and interactions between the TVCs and the TICs (to assess differences in the magnitude of the TVC effect as a function of betweenperson characteristics such as gender or ethnicity). Taken together, models can be constructed that simultaneously evaluate within-person influences (via TVCs) and betweenperson influences (via TICs) on stability and change of the outcome over time.

\section{CAN GROWTH IN TWO CONSTRUCTS BE SIMULTANEOUSLY MODELED OVER TIME?}

Although the TVC model allows for covariates to change in value over time, it is assumed that the covariates themselves are not characterized by a systematic growth process. For example, say that the repeated outcome was reading ability, and the TVC was number of days of instruction that were missed in a given academic year. It would be reasonable to assume that days of instruction may influence reading ability at a given time point but that there is not a systematic growth process underlying days of instruction missed (that is, children would not be expected to show consistent developmental trends in days missed). However, say instead that the outcome was again reading ability, but the TVC is substance use; in this case, developmental theory would predict that the onset and escalation of substance use itself is characterized by some type of systematic growth function. If so, then the TVC model may be mis-specified and result in biased effects.

Both the multilevel and SEM growth frameworks can be expanded to allow for the simultaneous growth of two constructs over time, and this is commonly called a multivariate growth model (Bollen \& Curran, 2006, chap. 7; MacCallum, Kim, Malarkey, \& KiecoltGlaser, 1997; McArdle, 1988). Each construct can be characterized by a unique functional form (e.g., one may be linear, the other quadratic), and their relation is examined at the level of the growth factors (e.g., direct estimates of the relation between the intercepts and slopes within and across construct). Finally, these multivariate models can themselves be extended 
to include one or more TICs to predict the set of growth factors. There are several variations of the multivariate model that attempt to simultaneously examine bidirectional effects between two constructs both at the level of the growth trajectories and at the level of repeated measures. Two examples include the latent difference score model (McArdle, Ferrer-Caja, Hamagami, \& Woodcock, 2002) and the autoregressive latent trajectory model (Bollen \& Curran, 2004; Curran \& Bollen, 2001), although several other approaches exist as well. The systematic study of the bidirectional relation between two or more constructs is a topic of much ongoing research, so we can expect additional multivariate methods to become available soon.

\section{CAN GROWTH MODELS BE SIMULTANEOUSLY ESTIMATED WITHIN TWO OR MORE GROUPS?}

It is important to realize that when estimating the growth models described thus far, strong assumptions are made about the equivalence of the model parameters across all individuals within the sample (e.g., Bollen \& Curran, 2006, chap. 6). As a simple example, consider fitting a model to data that consist of responses from males and females. If an unconditional growth model is fitted to the pooled sample (i.e., the usual single-group analysis), it is explicitly assumed that all of the parameters that define the growth model are precisely equal for both gender groups. If gender differences were hypothesized, the growth model can easily be expanded to include gender as a time-invariant predictor; however, this only introduces differences in the conditional means of the growth factors (e.g., on average, males may start higher or lower compared with females and increase more or less steeply). Gender thus serves to shift the conditional means of the intercept and slope to higher or lower values, yet all other parameters that govern the model are assumed to be equal between the two groups.

Whereas in many situations these assumptions are perfectly reasonable, in others, they may be distinctly questionable. For example, a potential outcome of a treatment intervention is to decrease variability in the expression of certain behaviors within the treatment group but not the control group over time (e.g., an intervention designed to decrease antisocial behavior in preschool children will also likely decrease the variability of types of disruptive behavior in the children exposed to the treatment). If these estimates of variability are markedly different across groups, yet a model is fitted that assumes these to be the same, then biased parameter estimates are expected. Both the SEM and multilevel approaches address this issue through the simultaneous estimation of growth models across two or more groups in what are called multiple-groups growth models. If all model parameters are set equal across all groups, this is equivalent to estimating a single-group growth model. Alternatively, if all parameters are allowed to freely vary across all groups, this is equivalent to estimating a growth model within each group separately. The typical application will fall somewhere between these two extremes in which some parameters are equated and others are not. This framework provides yet another option for maximally understanding growth processes both within and across groups.

\section{WHAT IF THERE IS A POTENTIALLY IMPORTANT GROUPING VARIABLE THAT WAS NOT DIRECTLY OBSERVED?}

In the multiple-groups growth model described above, any grouping variable of interest must be directly observed within the data. That is, groups based upon biological sex, treatment condition, or ethnic heritage must be unambiguously identified for each observation in the data set. This group identification measure is used to assign each case to its associated group, and the growth models are then simultaneously fit to the set of groups. 
However, there may be situations in which it is hypothesized that two or more groups exist in the sample, yet the grouping variable was not directly observed. For example, when studying lifetime trajectories of delinquent behavior, developmental theory may dictate that specific subgroups exist that are indirectly defined by the pattern of behavior over development, and thus, group membership is not an observed variable in the data set (e.g., Moffitt, 1993). That is, there is some latent group that was not directly observed yet whose existence must be estimated from the characteristics of the data. There has been a flurry of recent developments in the estimation of models such as these, and a number of terms are used to describe these types of models. Examples include growth mixture models, latent class growth models, and semi-parametric groups-based trajectory models, among others (e.g., B. O. Muthén, 2004; B. O. Muthén \& Shedden, 1999; Nagin, 2005). These techniques are being applied with increasing frequency in many areas of developmental research including the study of criminology, alcohol use, parenting, and reading difficulties (e.g., Boscardin, B. Muthén, Francis, \& Baker, 2008; B. O. Muthén \& L. K. Muthén, 2000; Nagin \& Land, 1993; Stoolmiller, 2001). Importantly, a number of nontrivial differences exist across these various approaches, and care must be taken in selecting the optimal strategy for a given research application. Further, although growth mixture models are both intriguing from a theoretical perspective and powerful from an analytical one, a number of concerns have been identified about the use of these techniques in practice (e.g., Bauer, 2007; Bauer \& Curran, 2003, 2004). As with any statistical procedure, it must be clearly established that the growth mixture model is the most appropriate analytical approach available for testing the specific research hypotheses at hand.

\section{WHERE DO I GO FROM HERE?}

We hope that we have been able to help guide you through at least an initial foray into the exciting collection of growth curve models that can be used with great effectiveness in many areas of developmental research. A logical final question is: Where does one go from here? An initial step is to turn to existing written work in this area. First, there are a number of more pedagogically oriented papers that walk the reader through different aspects of the application and interpretation of growth models; examples include Curran (2000), Curran and Hussong (2002, 2003), Duncan and Duncan (2004), Preacher et al. (2008), Singer (1998), and Willett, Singer, and Martin (1998). Second, there are several recently published textbooks that cover more comprehensive aspects of these techniques; examples include Bollen and Curran (2006), Duncan et al. (2006), Hedeker \& Gibbons (2006), Raudenbush \& Bryk (2002), and Singer and Willett (2003). Finally, there are a growing number of quality applications of various types of growth models within the developmental sciences; several recent examples include Brown, Meadows, and Elder (2007), McCoach, O'Connell, Reis, and Levitt (2006), Owens and Shaw (2003), and Williams, Conger, and Blozis (2007). Next, there are many well-developed online resources available that provide fully worked examples with empirical data and associated computer code; specific Web site addresses come and go, so the best strategy is to enter relevant terms in any major search engine and proceed from there. Finally, there are an increasing number of workshops available around the country that are focused on the theory and application of growth modeling within the social sciences; again, specifics change with time, but a bit of careful online searching will provide a current summary of available workshops. And if all else fails, send one of us an email and we'll try to point you in the right direction.

\section{CONCLUSION}

We have only briefly touched on just a few of the many interesting topics associated with the potential for growth models to help us gain a better understanding of individual differences in developmental change. Important remaining issues include growth models 
with binary or discrete outcomes (e.g., Mehta et al., 2004), incorporating alternative metrics of time (e.g., Mehta \& West, 2000), using growth trajectories as predictors (e.g., B. O. Muthén \& Curran, 1997; Seltzer, Choi, \& Thum, 2003), estimating statistical power for growth models (e.g., B. O. Muthén \& Curran; L. K. Muthén \& B. O. Muthén, 2002), and the estimation of hybrid autoregressive and change score models (Bollen \& Curran, 2004; McArdle, 2001). Growth models offer a plethora of exciting opportunities for testing theoretically derived hypotheses in ways not previously possible. Despite the strength and flexibility of these methods, even greater care must be taken to ensure that the estimated growth model maximally corresponds to the underlying developmental theory (e.g., Curran $\&$ Willoughby, 2003). Any disjoint that exists between the theoretical model and the statistical model only serves to undermine our ability to draw empirically informed conclusions about our theory under study. Despite this caveat, growth models have a tremendous amount to offer to a broad array of developmental research endeavors and represent a powerful set of tools to help us continue to propel forward as a science.

\section{References}

Allison, PD. Missing data. Newbury Park, CA: Sage Publications, Inc; 2001.

Arbuckle, JL. Full information estimation in the presence of incomplete data. In: Marcoulides, GA.; Schumaker, RE., editors. Advanced structural equation modeling: Issues and techniques. Mahwah, NJ: Lawrence Erlbaum Associates; 1996. p. 243-278.

Bauer DJ. Estimating multilevel linear models as structural equation models. Journal of Educational and Behavioral Statistics. 2003; 28:134-167.

Bauer DJ. Observations on the use of growth mixture models in psychological research. Multivariate Behavioral Research. 2007; 42:757-786.

Bauer DJ, Curran PJ. Distributional assumptions of growth mixture models: Implications for overextraction of latent trajectory classes. Psychological Methods. 2003; 8:338-363. [PubMed: 14596495]

Bauer DJ, Curran PJ. The integration of continuous and discrete latent variable models: Potential problems and promising opportunities. Psychological Methods. 2004; 9:3-29. [PubMed: 15053717]

Bollen, KA. On the origins of latent curve models. In: Cudeck, R.; MacCallum, R., editors. Factor analysis at 100. Mahwah, NJ: Lawrence Erlbaum Associates; 2007. p. 79-98.

Bollen KA, Curran PJ. Autoregressive latent trajectory (ALT) models: A synthesis of two traditions. Sociological Methods and Research. 2004; 32:336-383.

Bollen, KA.; Curran, PJ. Latent curve models: A structural equation perspective. Hoboken, NJ: Wiley; 2006.

Bollen, KA.; Long, JS. Testing structural equation models. Newbury Park, CA: Sage; 1993.

Boscardin C, Muthén B, Francis D, Baker E. Early identification of reading difficulties using heterogeneous developmental trajectories. Journal of Educational Psychology. 2008; 100:192-208.

Brown JS, Meadows SO, Elder GH. Race-ethnic inequality and psychological distress: Depressive symptoms from adolescence to young adulthood. Developmental Psychology. 2007; 43:12951311. [PubMed: 18020812]

Browne, M.; du Toit, SHC. Models for learning data. In: Collins, L.; Horn, JL., editors. Best methods for the analysis of change. Washington, DC: American Psychological Association Press; 1991. p. 47-68.

Bryk AS, Raudenbush SW. Application of hierarchical linear models to assessing change. Psychological Bulletin. 1987; 101:147-158.

Coffman DL, Millsap RE. Evaluating latent growth curve models using individual fit statistics. Structural Equation Modeling. 2006; 13:1-27.

Cronbach LJ, Furby L. How should we measure 'change' — or should we? Psychological Bulletin. 1970; 74:68-80.

Cudeck R, Harring JR. Analysis of nonlinear patterns of change with random coefficient models. Annual Review of Psychology. 2007; 58:615-637. 
Curran, PJ. A latent curve framework for studying developmental trajectories of adolescent substance use. In: Rose, J.; Chassin, L.; Presson, C.; Sherman, J., editors. Multivariate applications in substance use research. Hillsdale, NJ: Erlbaum; 2000. p. 1-42.

Curran PJ. Have multilevel models been structural equation models all along? Multivariate Behavioral Research. 2003; 38:529-569.

Curran PJ, Bauer DJ, Willoughby MT. Testing and probing main effects and interactions in latent curve analysis. Psychological Methods. 2004; 9:220-237. [PubMed: 15137890]

Curran, PJ.; Bollen, KA. The best of both worlds: Combining autoregressive and latent curve models. In: Collins, LM.; Sayer, AG., editors. New methods for the analysis of change. Washington, DC: American Psychological Association Press; 2001. p. 105-136.

Curran, PJ.; Hussong, AM. Structural equation modeling of repeated measures data. In: Moskowitz, D.; Hershberger, S., editors. Modeling intra-individual variability with repeated measures data: Methods and applications. New York: Erlbaum Associates; 2002. p. 59-86.

Curran PJ, Hussong AM. The use of latent trajectory models in psychopathology research. Journal of Abnormal Psychology. 2003; 112:526-544. [PubMed: 14674867]

Curran PJ, Willoughby MT. Implications of latent trajectory models for the study of developmental psychopathology. Development and Psychopathology. 2003; 15:581-612. [PubMed: 14582933]

Duncan TE, Duncan SC. An introduction to latent growth curve modeling. Behavior Therapy. 2004; 35:333-363.

Duncan, TE.; Duncan, SC.; Strycker, LA. An introduction to latent variable growth curve modeling: Concepts, issues, and applications. 2. Mahwah, NJ: Lawrence Erlbaum Associates; 2006.

Heckman JJ. The common structure of statistical models of truncation, sample selection, and limited dependent variables and a simple estimator for such models. Annals of Economic and Social Measurement. 1976; 5:475-492.

Hedeker, D.; Gibbons, R. Longitudinal data analysis. New York: Wiley Inc; 2006.

Huttenlocher J, Haight W, Bryk A, Seltzer M, Lyons T. Early vocabulary growth: Relation to language input and gender. Developmental Psychology. 1991; 27:236-248.

Little, RJ.; Rubin, DB. Statistical analysis with missing data. New York: Wiley Inc; 1987.

MacCallum RC, Kim C, Malarkey W, Kiecolt-Glaser J. Studying multivariate change using multilevel models and latent curve models. Multivariate Behavioral Research. 1997; 32:215-253.

McArdle, JJ. Dynamic but structural equation modeling of repeated measures data. In: Nesselroade, JR.; Cattell, RB., editors. Handbook of multivariate experimental psychology. New York: Plenum; 1988. p. 561-614.

McArdle, JJ. A latent difference score approach to longitudinal dynamic structural analysis. In: Cudeck, R.; du Toit, SHC.; Joreskog, KG., editors. Structural equation modeling: Present and future. Lincolnwood, IL: Scientific Software; 2001. p. 341-380.

McArdle JJ. Latent variable modeling of differences in changes with longitudinal data. Annual Review of Psychology. 2009; 60:577-605.

McArdle JJ, Epstein D. Latent growth curves within developmental structural equation models. Child Development. 1987; 58:110-133. [PubMed: 3816341]

McArdle JJ, Ferrer-Caja E, Hamagami F, Woodcock RW. Comparative longitudinal structural analyses of the growth and decline of multiple intellectual abilities over the life span. Developmental Psychology. 2002; 38:115-142. [PubMed: 11806695]

McCoach DB, O'Connell AA, Reis SM, Levitt HA. Growing readers: A hierarchical linear model of children's reading growth during the first 2 years of school. Journal of Educational Psychology. 2006; 98:14-28.

Mehta PD, Neale MC, Flay BR. Squeezing interval change from ordinal panel data: Latent growth curves with ordinal outcomes. Psychological Methods. 2004; 9:301-333. [PubMed: 15355151]

Mehta PD, West SG. Putting the individual back into individual growth curves. Psychological Methods. 2000; 5:23-43. [PubMed: 10937321]

Meredith W, Tisak J. Latent curve analysis. Psychometrika. 1990; 55:107-122.

Moffitt TE. Adolescence-limited and life-course persistent antisocial behavior: A developmental taxonomy. Psychological Review. 1993; 100:674-701. [PubMed: 8255953] 
Muthén, BO. Latent variable analysis: Growth mixture modeling and related techniques for longitudinal data. In: Kaplan, D., editor. Handbook of quantitative methodology for the social sciences. Newbury Park, CA: Sage Publications; 2004. p. 345-368.

Muthén BO, Curran P. General longitudinal modeling of individual differences in experimental designs: A latent variable framework for analysis and power estimation. Psychological Methods. 1997; 2:371-402.

Muthén BO, Muthén LK. Integrating person-centered and variable-centered analyses: Growth mixture modeling with latent trajectory classes. Alcoholism: Clinical and Experimental Research. 2000; 24:882-891.

Muthén BO, Shedden K. Finite mixture modeling with mixture outcomes using the EM algorithm. Biometrics. 1999; 55:463-469. [PubMed: 11318201]

Muthén LK, Muthén BO. How to use a Monte Carlo study to decide on sample size and determine power. Structural Equation Modeling. 2002; 9:599-620.

Nagin, DS. Group-based modeling of development. Cambridge, MA: Harvard University Press; 2005.

Nagin D, Land KC. Age, criminal careers, and population heterogeneity: Specification and estimation of a nonparametric, mixed Poisson model. Criminology. 1993; 31:327-362.

Nesselroade, JR. The warp and the woof of the developmental fabric. In: Downs, R.; Liben, L.; Palermo, DS., editors. Visions of aesthetics, the environment, \& development: The legacy of Joachim F. Wohlwill. Hillsdale, NJ: Lawrence Erlbaum Associates; 1991. p. 213-240.

Owens EB, Shaw DS. Predicting growth curves of externalizing behavior across the preschool years. Journal of Abnormal Child Psychology. 2003; 31:575-590. [PubMed: 14658739]

Preacher, KJ.; Wichman, AL.; MacCallum, R.; Briggs, NE. Latent growth curve modeling. Thousand Oaks, CA: Sage Publications; 2008.

Raudenbush, SW. Toward a coherent framework for comparing trajectories of individual change. In: Collins, L.; Sayer, A., editors. Best methods for studying change. Washington, DC: The American Psychological Association; 2001. p. 33-64.

Raudenbush, SW.; Bryk, AS. Hierarchical linear models: Applications and data analysis methods. 2. Thousand Oaks, CA: Sage Publications; 2002.

Rogosa D. A critique of cross-lagged correlation. Psychological Bulletin. 1980; 88:245-258.

Rogosa D, Willett JB. Understanding correlates of change by modeling individual differences in growth. Psychometrika. 1985; 50:203-228.

Rubin, DB. Multiple imputation for nonresponse in surveys. New York: Wiley Inc; 1987.

Satorra A. Robustness issues in structural equation modeling: A review of recent developments. Quality \& Quantity. 1990; 24:367-386.

Schafer, JL. Analysis of incomplete multivariate data. London: Chapman \& Hall; 1997.

Schafer JL. Multiple imputation: A primer. Statistical Methods in Medical Research. 1999; 8:3-15. [PubMed: 10347857]

Schafer JL, Graham JW. Missing data: Our view of the state of the art. Psychological Methods. 2002; 7:147-177. [PubMed: 12090408]

Seltzer M, Choi K, Thum YM. Examining relationships between where students start and how rapidly they progress: Using new developments in growth modeling to gain insight into the distribution of achievement within schools. Educational Evaluation and Policy Analysis. 2003; 25:263-286.

Singer JD. Using SAS PROC MIXED to fit multilevel models, hierarchical models, and individual growth models. Journal of Educational and Behavioral Statistics. 1998; 23:323-355.

Singer, JD.; Willett, JB. Applied longitudinal data analysis: Modeling change and event occurrence. New York: Oxford University Press; 2003.

Stoolmiller M. Synergistic interaction of child manageability problems and parent discipline tactics in predicting future growth in externalizing behavior for boys. Developmental Psychology. 2001; 37:814-825. [PubMed: 11699755]

Willett JB, Sayer AG. Using covariance structure analysis to detect correlates and predictors of individual change over time. Psychological Bulletin. 1994; 116:363-381. 
Willett JB, Singer JD, Martin NC. The design and analysis of longitudinal studies of development and psychopathology in context: Statistical models and methodological recommendations.

Development and Psychopathology. 1998; 10:395-426. [PubMed: 9635230]

Williams ST, Conger KJ, Blozis SA. The development of interpersonal aggression during adolescence: The importance of parents, siblings, and family economics. Child Development. 2007; 78:15261542. [PubMed: 17883446]

Wohlwill JF. Relations between method and partial-isomorphism view. Annals of Theoretical Psychology. 1991; 7:91-138. 